\title{
Blazar jet emission: a statistical approach
}

\section{Barbara Biasuzzi*}

INFN-Trieste and Udine University, via delle Scienze 206, I-33100 Udine, Italy

E-mail: barbara.biasuzzi@uniud.it

\section{Michele Peresano}

Udine University, via delle Scienze 206, I-33100 Udine, Italy

E-mail: peresano.michele@gmail.com

\section{Domenico Ferigo}

Email: ilpupalibero.it

\section{Stefano Ansoldi}

INFN-Trieste and Udine University, via delle Scienze 206, I-33100 Udine, Italy

E-mail: ansoldiefulbrightmail.org

\section{Massimo Persic}

INFN-Trieste and INAF/Osservatorio Astronomico di Trieste, via G.B.Tiepolo 11, I-34143

Trieste, Italy

E-mail: persiceoats.inaf.it

\begin{abstract}
Blazars belong to the class of active galaxies that host an active galactic nucleus (AGN) in their center and show a luminosity that far exceeds the luminosity of the host galaxy. The emission from blazar jets is strongly variable and non-thermal. Its spectral energy distribution (SED) is characterized by two humps located, respectively, at IR-X-ray and MeV-TeV frequencies. By modelling and fitting observational blazar SEDs we can derive the physical properties of these sources and better understand the processes that occur at very high energies. Even though several models have been proposed to describe blazar emission, so far SED models have been obtained only (mostly) heuristically. In this article we present a statistically rigorous approach and its implementation to efficiently rule out models for specific sources - when simultaneous datasets are available. We will also digress on some still unsolved technical problems that may appear when dealing with multi-frequency datasets, and report on recent progress to solve them.
\end{abstract}

Science with the New Generation of High Energy Gamma-ray experiments, 10th Workshop - Scineghe2014 04-06 June 2014

Lisbon - Portugal

\footnotetext{
* Speaker.
} 


\section{Introduction}

Active galactic nuclei (AGNs) are galactic nuclei whose luminosities far exceed the luminosities of their hosting galaxies. The engines of these sources are thought to be supermassive black holes that accrete matter from their surroundings. The accretion process is mediated by an accretion disk that dissipates angular momentum. Occasionally, two highly collimated relativistic jets shoot out perpendicular to the disk plane. AGNs look different and show different features depending on their orientation with regard to the observer: in particular AGNs whose jets point at the observer are called blazars. Blazars are interesting sources: they appear as the most powerful members of the AGN family, because their beamed emission - as seen by an observer sitting on the jet axis is amplified by the jet's relativistic boosting: $L \sim L_{0} \Gamma^{4}$ (where $\Gamma$ is the jet's Lorentz factor and $L$, $L_{0}$ are the observed and intrinsic luminosities, respectively). Relativistic beaming acts as a natural magnifying lens on the emission processes in the jet, whereas any emission from the host galaxy gets swamped out. For a complete review about AGNs features and properties see [1].

AGNs are characterized by a strongly variable non-thermal emission [2-4] whose spectral energy distribution (SED) is characterized by two peaks: one, located at low energies (IR to X-ray range), is thought to arise from synchrotron emission; the other, located at high energies ( $\mathrm{MeV}-\mathrm{TeV}$ range), is usually interpreted as Comptonized emission from some local photon field off relativistic electrons. Both peaks are required to sample the SED, because the synchrotron emissivity is degenerate in magnetic field and electron density: $j_{s} \sim n_{e} B^{(q+1) / 2}$ [where $n_{e}$ is the non-thermal electron density, $B$ the magnetic field intensity, and $q$ the power-law index of the electron spectrum, linked to the observed radio slope $\alpha$, by $\alpha=(q-1) / 2$ ]. Knowledge of the Compton peak provides the second equation $j_{I C} \sim n_{e} j_{s}$, needed to remove the degeneracy.

Several hadronic [5, 6] and leptonic models have been proposed to explain blazar emission, but the latter are the more commonly used ones. Among them, most popular are (i) the one-zone and two-zones Synchrotron-Self-Compton (SSC) models [7] (Comptonization of synchrotron radiation off same parent electrons), and (ii) the External Compton (EC) models [8] (Comptonization of radiation from either the disk or other regions outside the jet).

\section{Fitting Spectral Energy Distributions}

In this contribution we focus on a fairly common and simple way to describe blazar emission, through an SSC model characterized by at least seven free parameters. Although historically it was standard practice to determine their best (i.e., most likely) values using an eye-ball comparison of the data with the chosen model, here we will consider more recent approaches attempting to perform fits using $\chi^{2}$ minimization [9]. The number of free parameters also depends on the assumed electron spectrum: seven parameters are necessary when the emitted electrons are distributed as a simple power-law spectrum. In particular, some of these parameters can model phenomenological features of the spectrum, but we think it is important to connect them with the underlying physics. With reference to the results in $[10,11], \chi^{2}$ minimization has been achieved with standard techniques, i.e., the Levenberg-Marquardt method [12], an efficient approach that combines the steepest-descent method [13] and the inverse-Hessian method [14]. 
As is well known, at each step the algorithm will need to compute the model function and the derivatives of the model function with respect to the model parameters. For the model considered in $[10,11]$ the SED for the chosen SSC model could be obtained only as a numerical sample, that was calculated within a proprietary framework: this turned out to be the most demanding step in terms of computational time. Details of this approach can be found in [15].

Once the best values of the model parameters have been obtained by non-linear $\chi^{2}$ minimization, the Kolmogorov-Smirnov test was performed as a goodness-of-fit test [16]. In all cases considered the test failed at 5\% significance level: it was argued that a possible reason for that failure could be that two distinct physical processes manifested themselves at very different energies, and/or that the uncertainties associated with the very-high-energy data are much larger than those associated with optical and X-ray data. A conclusive result, however, was not reached, so (a combination of these and) other possibilities should be seriously considered as well.

\subsection{The cases of Mrk 421 and Mkn 501}

The SSC parameter values obtained with the $\chi^{2}$ minimization are generically consistent with those expected for high-frequency BL Lac objects [10, 11].

The Mkn 421 paper [10] was substantially a feasibility test. The goal was to show that a rigorous statistical approach to multi-frequency data fitting was already possible back then, and that meaningful physical results could be obtained. The reason why such an outcome was not trivially expected was a not only that the model had a complex non-linear dependence from a relatively large number of parameters, but also that the data sets were naturally inhomogeneous: they stretched over several decades in frequency, and according to the frequency ranges, they came from different experimental techniques, with different systematics and data analysis methods. This was not exactly a typical situation, and the Mkn 421 paper emphasized the necessity for refinements, which especially appeared in the failure of a standard, distribution independent, goodness-of-fit test and in the very large uncertainties associated to some of the parameters. Especially the latter issue was particularly unwelcome, sometimes reducing the predictive power of the method to the identification of upper limits only. With reference to what follows, we would like to also emphasize that this very same issue made the discussion about the choice of the starting point in parameter space purely academic, as consistency among different choices with large error bars on the parameters was clearly and easily guaranteed.

The large error bars obtained in the work on Mkn 421 suggested a closer analysis of the issue in a follow-up paper, on Mkn 501 [11]. For instance one could think that the quadratic approximation to the $\chi^{2}$ surface could be not accurate enough, resulting in an overstimation of the uncertaintes. Unanticipated correlations among the parameters, and/or an inaccurate identification of the $\chi^{2}$ minimum, might also manifest themselves in a wrong estimate of the uncertainties.

A more in-depth study of the $\chi^{2}$ function around the obtained minimum was then performed while studying Mkn 501. It turned out that the uncertainties obtained with the standard textbook approach, that considers a quadratic approximation to the $\chi^{2}$ hypersurface were much larger than those (obtained) by a direct study of $\chi^{2}$ around the obtained minimum. However, this potential improvement was offset by the fact that, because of the much smaller uncertainties, stability in determining of the minimum $\chi^{2}$ versus the starting point in parameter space was more challenging 
to attain. Some suspiciously low $\chi^{2}$-values suggested, too, a closer scrutiny of the shape of the $\chi^{2}$ manifold near its minimum.

\section{Progress report}

An immediate idea that comes to mind in order to tackle the difficulties discussed above is to perform a more dense sampling of $\chi^{2}$ in parameter space (e.g., by repeating the $\chi^{2}$ minimization process from a large set of initial points). This would have requested much larger computational resources (hundreds or thousands of times more computational power) with the code used in the previously mentioned papers. It was then high time to improve code efficiency, which could be done by writing dedicated a $\mathrm{C} / \mathrm{C}++$ code to replace the SED generation routines that were written in a much slower proprietary framework. Preliminary tests showed that a gain of about a factor of 50 in the SED generation time could be achieved by an adapted $\mathrm{C}++$ code. With a 50 times faster code it would have been possible to drop the computational power requirements to the level of what could be run on an average workstation in about one day computation time, which was well within a standard individual computational framework. In what follows we detail some of the features of the new code which, after preliminary development began in 2012, is now in the final test stage:

1. we created a SED class with the following methods:

- synchrotron, which computes the synchrotron emission, including the synchrotron self-adsorption;

- compton, which computes the emission due to inverse Compton, calculating the emissivity spectrum and the specific power emitted per frequency unit;

— ssc, which computes the final emissivity considering, both, synchrotron and Compton contributions.

2. other $\mathrm{C}++$ classes have been added, e.g., to compute astronomical distances;

3. dedicated methods have been coded implementing standard algorithms for integration and derivation as well as for data file manipulation;

4. we created a MINIMIZATION class containing an ad hoc modified version of the LevenbergMarquardt minimization method given in [17] whose member function also contains the minimization algorithm already used in $[10,11]$.

Preliminaries tests have shown that:

1. with the new code consistency with different choices of the starting point in parameters space can usually be achieved;

2. uncertainties obtained with a detailed analysis of the $\chi^{2}$ hypersurface result in a higher predictive power of the fitted parameters; a higher consistency with uncertainties obtained by the standard textbook approach is also usually achieved. 
The above can be interpreted as a more accurate determination of the $\chi^{2}$ minimum and, thus, of the parameters values, which could not be achieved at the precision level that could be reached with the slower code because of lack of computational power. However, we can anticipate that in turn this also results in smaller values of $\chi^{2}$ : these are sometimes smaller than expected, which seems to point suggest a possible overestimation of the uncertainties associated to some of the observed points ${ }^{1}$. Based on the status that we are reporting here, we are now planning to:

1. implement other leptonic models such as the two-zone SSC and the EC model;

2. allow the user to choose among different initial electron spectra;

3. apply the minimization process on artificial data to test the efficiency of the algorithm.

\section{Conclusion}

Blazar SED models were traditionally obtained by a heuristic approach, mainly due to severe lack of simultaneous multi-frequency data. This makes it difficult to extract significance from the results in a statistically meaningful way. It might also make challenging the comparison of results coming from different analyses. Nevertheless, in recent years simultaneous multi-frequency campaigns have provided a broad-band coverage of source activity and many data sets have become available, so that emission modelling can be attempted more successfully. Here we have briefly discussed a recent proposal for a standardized approach to a rigorous, statistical analysis aimed at estimating the emission parameters of a specific SSC model. Starting from the pros and cons of the method described in Section 2, we have then discussed recent work in the development of a consistent computational framework to address this problem, and perspectives for future development.

As anticipated by the work in [10,11], this approach has the potential to provide results with a clear statistical meaning. In particular, we are planning: (i) to optimize the code in order to reduce computational time and better estimate the model parameters and their uncertainties; (ii) to implement different leptonic models (in particular the two-zone SSC and EC models), allowing the user to choose among different electron spectra to efficiently rule out some specific models for a given source; (iii) to carry out a statistical study on blazar properties based on homogeneous analyses of large, available data sets.

\section{References}

[1] B. M. Peterson, An introduction to active galactic nuclei, Cambridge University Press, Cambridge 1997.

[2] L. Maraschi, C. Ghisellini, A. Celotti, A jet model for the gamma-ray emitting blazar 3C 279, ApJ 395 (1992) L5.

[3] F. Tavecchio, L. Maraschi, G. Ghisellini, Constraints on the physical parameters of TeV blazars, ApJ 509 (1998) 608, [arXiv: astro-ph/9809051].

[4] G. Ghisellini et al., High-redshift Fermi blazars, MNRAS 411 (2011) 901, [arXiv: 1009.3275$].$

\footnotetext{
${ }^{1}$ Work in this respect is at a very preliminary stage and the qualitative result reported here should be taken with great care. We will report more details about this in later work after collecting more evidence.
} 
[5] W. Bednarek, On the gamma-ray emission from 3C 279, ApJ 402 (1993) L29.

[6] K. Mannheim, The proton blazar, A\&A 269 (1993) 67, [arXiv : astro-ph/9302006].

[7] F. Tavecchio et al., TeV BL Lac objects at the dawn of the Fermi era, MNRAS 401 (2010) 1570, [arXiv:0909.0651].

[8] C. D. Dermer, R. Schlickeiser, Model for the high-energy emission from blazars, ApJ 416 (1993) 458.

[9] P. R. Bevington, D. K. Robinson, Data reduction and error analysis for the physical sciences, McGraw-Hill Edition, New York 1992.

[10] N. Mankuzhhiyil et al., The environment and distribution of emitting electrons as a function of source activity in Markarian 421, ApJ 733 (2011) 14, [arXiv: 1103.0909 ].

[11] N. Mankuzhhiyil et al., Emitting electrons and source activity in Markarian 501, ApJ 753 (2012) 154, [arXiv:1205.5237].

[12] D. W. Marquardt, An algorithm for least-squares estimation of nonlinear parameters, SIAM Journal on Applied Mathematics 11 (1963) 431.

[13] K. Madsen, N. B. Nielsen, O. Tingleff, Methods for non-linear least squares problems, Informatics and Mathematical Modelling, Technical University of Denmark 2004.

[14] A. Björck, Numerical methods for least squares problems, SIAM, Philadelfia 1963.

[15] S. Ansoldi, A statistical approach to the study of AGN emission versus activity (with the detailed analysis of Mrk421), in proceedings of IRAP Workshop "From Nuclei to White Dwarfs and Neutron Stars”, ed. A. Mezzacappa \& R. Ruffini (Singapore: World Scientific), in press, arXiv: 1201.2428.

[16] W. T. Eadie et al., Statistical methods in experimental physics, North Holland, Amsterdam 1971.

[17] W. H. Press et al., Numerical recipes in C, Cambridge University Press, New York 1992. 